\title{
Insulin resistance in severe acne vulgaris
}

\author{
Nazan Emiroğlư ${ }^{1}$, Fatma Pelin Cengiz ${ }^{1}$, Funda Kemeriz ${ }^{2}$
}

${ }^{1}$ Department of Dermatology, Bezmialem Vakif University, Istanbul, Turkey
Head of the Department: Prof. Nahide Onsun MD, PhD
2Department of Dermatology, Aksaray State Hospital, Aksaray, Turkey
Head of the Department: Dr. Cengiz Kilicaslan MD

Postep Derm Alergol 2015; XXXII (4): 281-285

DOI: 10.5114/pdia.2015.53047

\begin{abstract}
Introduction: Acne vulgaris is a pilosebaceous gland disease that usually affects people from puberty to young adulthood. It is seen especially on the face, neck, trunk and arms. Its severity differs from patient to patient and its pathogenesis is multifactorial. The main pathogenic factors of acne are high sebaceous gland secretion, follicular hyperproliferation, high androgen effects, propionibacterium acnes colonization and inflammation. Diet is always thought a probable reason for acne and many studies are done about acne and diet.

Aim: To determine the effect of insulin resistance in severe acne vulgaris.

Material and methods: Two hundred and forty-three acne vulgaris patients and 156 healthy controls were enrolled into the study. The blood levels of insulin and glucose were measured. Homeostasis Model Assessment (HOMA) Index was calculated. The values were compared with the control group.

Results: All of the patients were in the severe acne group according to their scores on the global acne scoring scale. While fasting blood glucose levels were not different between the groups ( $p>0.05,82.91 \pm 9.76$ vs. $80.26 \pm 8.33$ ), the fasting insulin levels were significantly higher in the patient group than in the control group $(p<0.001,14.01$ \pm 11.94 vs. $9.12 \pm 3.53$ ). Additionally, there was a highly significant difference between the patient and control groups in terms of HOMA values ( $p<0.001,2.87 \pm 2.56$ vs. $1.63 \pm 0.65$ ).
\end{abstract}

Conclusions: These results suggest that insulin resistance may have a role in the pathogenesis of acne.

Key words: acne, diet, insulin, insulin resistance.

\section{Introduction}

Acne vulgaris is a common pilosebaceous gland disease that is most commonly seen during adolescence. Its severity differs from individual to individual [1]. Four major factors play roles in the pathogenesis of acne vulgaris: hyperplasia of sebaceous glands and increased sebum production, hyperkeratinization of pilosebaceous ducts, Propionibacterium acnes colonization and periglandular dermal inflammation [2]. Androgens stimulate the production of sebum, growth of the sebaceous glands and hyperkeratinization. Recent studies have shown that diet could play a role in the pathogenesis of acne [3, 4]. It has also been shown that a low glycemic index (GI) diet induced an improvement in acne severity and a parallel improvement in insulin sensitivity in young males with acne vulgaris.

Therefore, insulin and carbohydrate metabolism may have a role in the etiology and severity of the acne [5].

\section{Aim}

The aim of this study was to investigate the presence of insulin resistance in patients with severe acne vulgaris.

\section{Material and methods}

Two hundred and forty-three patients clinically diagnosed with severe acne vulgaris and 156 healthy controls were enrolled in the study. Informed consent was received from all participants and the Ethics Committee approved the study. A detailed history was taken from each participant and physical examinations were performed. The participants were excluded if they reported medications known to affect insulin metabolism, previous treatment with oral retinoids, hormone treatment for any reason in the previous 3 months, cigarette smoking, thyroid dysfunction, or a history of diabetes mellitus, hypertension, atherosclerotic vascular disease, malignancy,

Address for correspondence: Nazan Emiroğlu PhD, Department of Dermatology, Bezmialem Vakif University, Istanbul, Turkey, phone: +90 5057424786, e-mail: dr.nazanyilmaz@hotmail.com Received: 26.07.2014, accepted: 12.11.2014. 
Table 1. Global acne grading system

\begin{tabular}{ll}
\hline Location & Factor \\
\hline Forehead & 2 \\
\hline Right cheek & 2 \\
\hline Left cheek & 2 \\
\hline Nose & 1 \\
\hline Chin & 1 \\
\hline Chest and upper back & 3
\end{tabular}

Each type of lesion is given a value depending on severity: no lesions $=0$, comedones $=1$, papules $=2$, pustules $=3$ and nodules $=4$. The score for each area (Local score) is calculated using the formula: local score $=$ factor $\times$ grade $(0-4)$. The global score is the sum of local scores, and acne severity was graded using the global score. A score of 1-18 is considered mild; 19-30, moderate $31-38$, severe; and $>39$, very severe.

pregnancy or any other systemic inflammatory diseases. A total of 156 healthy adolescents underwent a detailed clinical, biochemical and hormonal evaluation and were found to be free of any systemic illness. The severity of the patients' acne was evaluated using the Global Acne Grading System (Table 1). Only patients whose global acne scores were moderate, severe or very severe were included in the study. Body mass index was calculated using Quetelet index (weight/height ${ }^{2}, \mathrm{~kg} / \mathrm{m}^{2}$ ). The World Health Organization's classification for obesity of body mass index (BMI) values $\geq 30 \mathrm{~kg} / \mathrm{m}^{2}$ was used.

Peripheral venous blood samples were collected from participants after $10 \mathrm{~h}$ of fasting. The blood samples were held at room temperature for 30 min after which they were centrifuged at 4000 rpm for 5 min to isolate the serum. Fasting blood glucose was determined by the hexokinase method and fasting insulin levels were measured using a commercial kit from the Diagnostic Products Corporation (Los Angeles, CA). Homeostatic model assessment (HOMA-IR) values, which indicate insulin resistance, were calculated using the following formula: fasting insulin levels $(\mu \mathrm{U} / \mathrm{ml}) \times$ fasting glucose $(\mathrm{mmol} / \mathrm{l}) / 22.5$. Values over 2.7 were interpreted as indicating insulin resistance.

\section{Statistical analysis}

All statistical calculations were performed using the Statistical Package for Social Sciences (SPSS) v15.0 for Microsoft Windows. Student $t$-tests and Mann-Whitney $U$ tests were used for comparing continuous variables. The $\chi^{2}$ test and Fisher's Exact test were used for categorical variables. Calculated $p$-values less than 0.05 were considered significantly different. Calculated $p$-values less than 0.01 were considered highly significantly different.

\section{Results}

Two hundred and forty-three patients with severe acne vulgaris and 156 healthy controls were included in the study. Two hundred and forty-three severe acne vulgaris patients (144 females, 59.3\%; 99 males, 40.7\%) and 156 healthy controls (111 females; $71.2 \%, 45$ males; $28.8 \%$ ) participated in the study. There was no significant difference between the patient and control groups with respect to sex or age $(p>0.05)$. The patient group's ages ranged from 14 to 35 years (mean age: $19.94 \pm 4.77$ ). The control group's ages were also 14 to 35 (mean age: 19.22 \pm 0.69 ). There was no difference between two groups in terms of weight, height or body mass index (all $p$ values $>0.05$ ). All of the patients were in the severe acne group according to their scores on the global acne scoring scale. While fasting blood glucose levels were not different between the groups ( $p>0.05,82.91 \pm 9.76$ vs. $80.26 \pm 8.33$ ), the fasting insulin levels were significantly higher in the patient group than in the control group $(p<0.001,14.01$ \pm 11.94 vs. $9.12 \pm 3.53$ ). Additionally, there was a highly significant difference between the patient and control groups in terms of HOMA values ( $p<0.001,2.87 \pm 2.56$ vs. $1.63 \pm 0.65$ ). The results of the statistical analyses comparing the patient and control groups are summarized in Table 2 and Figure 1.

Table 2. The results of the statistical analyses comparing the patient and control groups

\begin{tabular}{|c|c|c|c|}
\hline Features & $\begin{array}{c}\text { Patients }(n=243) \\
\text { Mean } \pm \text { SD }\end{array}$ & $\begin{array}{c}\text { Controls }(n=156) \\
\text { Mean } \pm \text { SD }\end{array}$ & Value of $p$ \\
\hline Gender (male/female) & $0.41 \pm 0.49$ & $0.29 \pm 0.45$ & $<0.05$ \\
\hline Age [year] & $19.94 \pm 4.77$ & $19.22 \pm 0.69$ & $<0.05$ \\
\hline Weight [kg] & $63.86 \pm 11.32$ & $61.96 \pm 11.54$ & $<0.05$ \\
\hline Height $[\mathrm{cm}]$ & $167.56 \pm 8.30$ & $166.4 \pm 7.09$ & $<0.05$ \\
\hline BMI $\left[\mathrm{kg} / \mathrm{m}^{2}\right]$ & $22.6 \pm 2.95$ & $22.3 \pm 3.52$ & $<0.05$ \\
\hline Insulin $[\mu \mathrm{U} / \mathrm{ml}]$ & $14.01 \pm 11.99$ & $9.12 \pm 3.55$ & $<0.001$ \\
\hline Glucose $[\mathrm{mmol} / \mathrm{l}]$ & $82.91 \pm 9.8$ & $80.26 \pm 8.385$ & 0.111 \\
\hline HOMA & $2.87 \pm 2.572$ & $1.63 \pm 0.663$ & $<0.001$ \\
\hline
\end{tabular}




\section{Discussion}

Acne is a widespread and complex skin disease affecting individuals of all ages, particularly between the ages of 15 to 17 years. In Western populations, 79-95\% of the adolescents is affected [6]. Acne is also seen into adulthood [7].

Although hyperseborrhea, follicular hyperkeratinization, Propionibacterium acnes colonization and inflammation are found to be responsible in the pathogenesis, the mechanism of acne is not known clearly [8]. The relationship between diet and acne has been uncertain in recent years. The studies have been conducted over the years to support this hypothesis but they have yielded contradictory results. Therefore, in this study, we aim at investigating the relationship between severe acne vulgaris and insulin resistance.

The evidence suggests that high glycemic load (HGL) diets may trigger acne by inducing hyperinsulinemia [912]. Low glycemic load (LGL) diets may play a dual role in the prevention of hyperinsulinemia by lowering the postprandial insulin [9-12]. Recent reports have shown that high intakes of refined, high-GI carbohydrates may be a significant cause of acne in Western countries [6].

Other reports have suggested that milk consumption may be a cause for acne outbreaks. Although milk has a low glycemic index, it could aggravate acne by increasing the levels of insulin like growth factor-1 (IGF-1). This effect was seen with fat-free milk consumption (but not the fat portion of milk) because of the presence of IGF in the nonfat portion of milk and IGF has effects on keratinocyte proliferation and apoptosis $[13,14]$. Milk also contains comedogenic hormones such as estrogen, progesterone, androgen precursors and $5 \alpha$-reductase steroids [15]. Another clinical example about the relation between acne and IGF is Laron syndrome (LS). Laron syndrome is characterized by congenital IGF-1 deficiency. In his study, Ben-Amitai and Laron observed that IGF-1 deficiency prevents the occurrence of acne. They suggested that IGF-1 and androgens are necessary for development of acne [16].

One of the most common questions regarding diet and acne is "Does chocolate cause acne?" With regard to this subject, Anderson performed a study by giving patients increasing amounts of chocolate day by day and observed that acne flared up with an increased consumption of chocolate [17]. In another study from Australia, plasma was examined in participants after diets with or without chocolate. As a result, postprandial hyperinsulinemia (28\% of patients) was seen in chocolate-fed participants, especially in patients who consumed chocolate milk instead of plain milk. The highest levels were observed with consumption of chocolate milk (48\% higher than plain milk) and dark chocolate milk in comparison with plain milk (13\% higher) [18]. Other studies have concluded that amino acids found in chocolate (e.g. arginine, leucine, phenylalanine) are quite insulinotropic

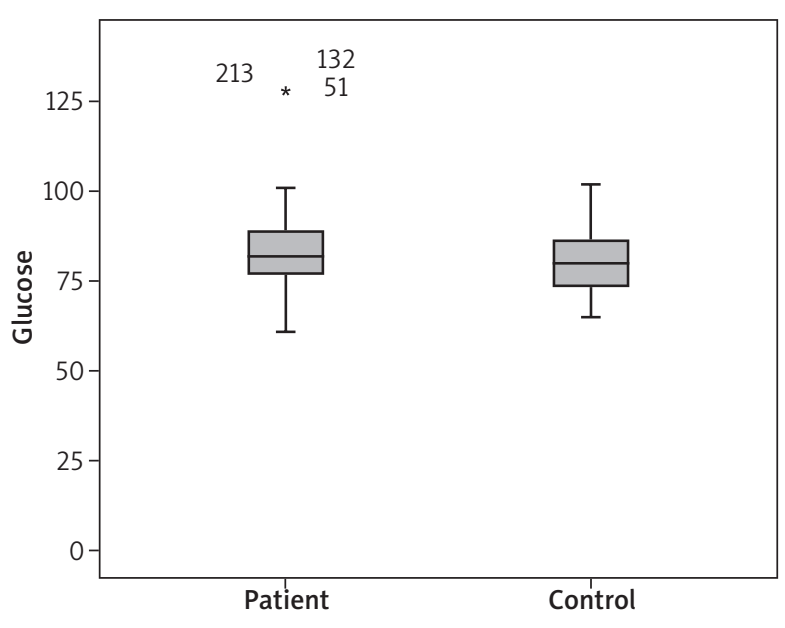

Figure 1. The Box-plot graphic of the glucose levels in patient and control groups

$[19,20]$. Chocolate also contains caffeine, theobromine, serotonin, phenylethylamine, triglycerides, and cannabinoid-like fatty acids that induce insulin secretion and insulin resistance $[21,22]$. Chocolate consumption as part of the typical Western diet may have played a role in the results of our current study.

As a result, diet can cause acne via insulin metabolism. Androgens, estrogens, progesterone, growth hormone, insulin, IGF-1, corticotropin-releasing hormone, adrenocorticotropic hormone, melanocortins, and glucocorticoids are related to acne [23-25].

Some chronic diseases support the relationship between acne and insulin metabolism. Polycystic ovary syndrome (PCOS) is one of them, where obesity, hyperinsulinemia, insulin resistance and hyperandrogenism are commonly observed. Acne is also a common occurrence in PCOS patients, as are high levels of IGF-1 and androgens. Both insulin and IGF induce the production of androgens while simultaneously inhibiting the hepatic synthesis of sex hormone-binding globulin (SHBG), therefore the bioavailability of androgens increases. The comedogenic effects of IGF-1 and high androgen levels are thought to be responsible for the acne seen in PCOS [3]. Hyperinsulinemia increases serum levels of IGF-1 and reduces serum levels of insulin-like growth factor binding protein 3 (IGFBP3). These two factors directly influence proliferation and apoptosis of keratinocytes [26]. Insulin-like growth factor may also stimulate some comedogenic factors such as androgens, growth hormone, and glucocorticoids $[13,14,27]$.

Treatments such as acarbose and metformin reduce insulin secretion and/or increase insulin sensitivity. These drugs have been demonstrated to reduce serum androgen levels and gonadotropins, and to improve acne and hirsutism, the menstrual cycle, ovulation, and fertil- 
ity in PCOS $[24,25]$. Sebum production starts at puberty. Acne firstly appears when plasma insulin, body mass index (BMI), GH and IGF-1 concentrations increase. The incidence of acne corresponds more closely to insulin and IGF-1 levels than plasma androgens $[4,25,28]$.

Insulin resistance may occur during various clinical conditions such as physiological conditions (e.g. puberty, pregnancy, old age, physical inactivity), as a side effect of certain medications (e.g. corticosteroids, some oral contraceptives, diuretics), or as a symptom of certain diseases (e.g. type 2 diabetes, cardiovascular disease, essential hypertension, PCOS, nonalcoholic fatty liver disease, certain cancers, sleep apnea syndrome) [29]. Given that the average age of the patients enrolled in the current study was nineteen, it is unlikely these chronic clinical conditions were the cause of their insulin resistance. It is more likely that their dietary habits caused their insulin resistance; however, dietary habits were only investigated verbally. In a previous study, the correlation between the typical Western diet and acne was investigated. Three non-Western societies were evaluated (Papua New Guinea, the Ache Indians of Paraguay, and the Natives of Kitava Island). Acne was not seen in these populations and their diets were cited as the likely explanation. Their diet includes lower glycemic index foods in comparison with Western diets [6]. According to these findings, researchers suggested that carbohydrate intake, especially in conjunction with a high glycemic index, could induce excessive secretion of insulin. As a result, insulin would affect follicular keratinization and sebaceous secretion [6]. The results of a recent study by Kaymak et al. suggest that the glycemic index of a diet and postprandial insulin levels do not play roles in the pathogenesis of acne, however this study is the only one that contradicts other studies [30]. The dietary habits of the modern Turkish population resemble those of the West. This resemblance is higher in students because of their relatively high consumption of fast food. In our study, serum insulin levels and HOMA-IR index values in patients with severe acne were higher than those of the control group. One reason for this result may be the consumption of high glycemic index diets, and our study is in support of the majority of studies which have found a positive link between diet and insulin, and acne.

In our study, there was no difference between two groups in terms of BMI. However, Del Prete et al. recently provided evidence that young Italian males affected with acne had a high BMI and exhibited insulin resistance [28]. Acne is also an important indicator on systemically overdone MTORC1 signaling that cause metabolic deviation on the way to serious mTORC1-driven diseases of civilization, especially obesity, arterial hypertension, insulin resistance, type 2 diabetes mellitus, cancer, and Alzheimer's disease [31]. So it is suggested that we should not only focus on the treatment of acne's skin pathology, but should appreciate the great chance for dietary intervention [31].

In our study we used HOMA-IR index for evaluating insulin resistance. The HOMA-IR index has been an accepted formula for measuring insulin resistance since its original publication by Matthews et al. in 1985 [32]. There are many various methods to describe insulin sensitivity. Among other models, the gold standard methods are euglycemic clamp and modified minimal model but they are used for research only because they are too invasive for general epidemiologic studies [33, 34]. In fact there is no need for intravenous access, the oral glucose tolerance test (OGTT) is appropriate for the assessment of large populations. Although OGTTs are more difficult to measure fasting glucose and insulin levels, the OGTT has minimal-risk for individual subjects [35]. However, the use of OGTT in large populations is limited. Therefore, methods such as a fasting insulin level, fasting glucose/ insulin ratio (FGIR), homeostasis model assessment for insulin resistance (HOMA-IR) and quantitative insulin-sensitivity check index (QUICKI) are suggested to be used in population studies [36]. HOMA-IR is a frequently used parameter in clinical research [36].

Although this validated method is used in many different countries for evaluating insulin resistance, cut-off points vary from country to country [37]. Recently, the cut-off value of HOMA-IR was determined at 2.7 in a Brazilian study [37], which is the same value that is accepted according to the Turkish Metabolic Syndrome Guides [38]. In our study, we chose to evaluate insulin resistance according to the Turkish Metabolic Syndrome Guides so our patients were diagnosed as insulin resistance according to the cut-off value of 2.7. In our study we observed a positive correlation between insulin resistance and severe acne vulgaris because there was a highly significant difference between the patient and control groups in terms of HOMA values ( $p<0.001,2.87 \pm 2.56$ vs. 1.63 $\pm 0.65)$.

\section{Conclusions}

We found a positive correlation between insulin resistance and severe acne vulgaris. These findings suggest that treatments prescribed for insulin resistance are worth investigating for treatment of severe acne vulgaris.

\section{Conflict of interest}

The authors declare no conflict of interest.

\section{References}

1. Cunliffe WJ, Simpson NB. Disorders of the sebaceous gland. In: Textbook of dermatology. $7^{\text {th }}$ ed. Burns T, Breathnach S, Cox N, Griffiths C (eds). Blackwell Science, Massachusetts 2004; 43.1-43.75. 
2. Zouboulis CC, Eady A, Philpott M, et al. What is the pathogenesis of acne? Exp Dermatol 2005; 14: 143-52.

3. Cordain L. Implications for the role of diet in acne. Semin Cutan Med Surg 2005; 24: 84-91.

4. Szyszkowska B, Łepecka-Klusek C, Kozłowicz K, et al. The influence of selected ingredients of dietary supplements on skin condition. Postep Derm Alergol 2014; 31: 174-81.

5. Smith RN, Mann NJ, Braue A, et al. A low-glycemic-load diet improves symptoms in acne vulgaris patients: a randomized controlled trial. Am J Clin Nutr 2007; 86: 107-15.

6. Cordain L, Lindeberg S, Hurtado M, et al. Acne vulgaris a disease of Western civilization. Arch Dermatol 2002; 138: 1584-90.

7. Law MP, Chuh AA, Lee A, Molinari N. Acne prevalence and beyond: acne disability and its predictive factors among Chinese late adolescents in Hong Kong. Clin Exp Dermaol 2010; 35: 16-21.

8. Bataille V, Snieder H, MacGregor AJ, et al. The influence of genetics and environmental factors in the pathogenesis of acne: a twin study of acne in women. J Invest Dermatol 2002; 119: 1317-22.

9. Willett W, Manson J, Liu S. Glycemic index, glycemic load and risk of type 2 diabetes. Am J Clin Nutr 2002; 76 (Suppl): 274S-80S.

10. Jenkins D, Kendall C, Augustin L, et al. Glycemic index: overview of implications in healthy and disease. Am J Clin Nutr 2002; 76 (Suppl): 266S-73S.

11. Carnethon MR, Loria CM, Hill JO, et al. Risk factors for the metabolic syndrome: the coronary artery risk development in young adults (CARDIA) study, 1985-2001. Diabetes Care 2004; 27: 2707-15.

12. McKeown N, Meigs J, Liu S, et al. Carbohydrate nutrition, insulin resistance and the prevalence of the metabolic syndrome in the Framingham offspring cohort. Diabetes Care 2004; 27: 538-46.

13. Adebamowo CA, Spiegelman D, Danby FW, et al. High school dietary dairy intake and teenage acne. J Am Acad Dermatol 2005; 52: 207-14.

14. Adebamowo CA, Spiegelman D, Berekey CS, et al. Milk consumption and acne in adolescents girls. Dermatol Online J 2006; $2: 1$.

15. Danby FW. Acne and milk, the diet myth, and beyond. J Am Acad Dermatol 2005; 52: 360-2.

16. Ben-Amitai D, Laron Z. Effect of insulin-like growth factor-1 deficiency or administration on the occurrence of acne. J Eur Acad Dermatol Venereol 2011; 25: 950-4.

17. Anderson PC. Foods as the cause of acne. Am Fam Physician 1971; 3: 102-3.

18. Brand-Miller J, Holt SH, de Jong V, et al. Cocoa powder increases postprandial insulinemia in lean young adults. J Nutr 2003; 133: 3149-52.

19. Van Haeften TW, Voetberg GA, Gerich JE, et al. Dose-response characteristics for arginine-stimulated insulin secretion in man and influence of hyperglycemia. J Clin Endocrinol Metab 1989; 69: 1059-64.

20. Van Loon LJ, Saris WH, Verhagen H, Wagenmakers AJ. Plasma insulin responses after ingestion of different amino acid or protein mixtures with carbohydrate. Am J Clin Nutr 2000; 72: 96-105.

21. Herraiz T. Tetrahydro-beta-carbolines, potential neuroactive alkaloids, in chocolate and cocoa. J Agric Food Chem 2000; 48: 4900-4.

22. Bruinsma K, Taren DL. Chocolate: food or drug? J Am Diet Assoc 1999; 99: 1249-56.
23. Capitanio B, Sinagra JL, Bordignon V, et al. Underestimated clinical features of post-adolescent acne. J Am Acad Dermatol 2010; 63: 782-8.

24. Melnik BC, Schmitz G. Role of insulin, insulin-like growth factor-1, hyperglycemic food and milk consumption in the pathogenesis of acne vulgaris. Exp Dermatol 2009; 1: 1-9.

25. Arora MK, Yadav A, Saini V. Role of hormones in acne vulgaris. Clin Biochem 2011; 44: 1035-40.

26. Treloar V, Logan AC, Danby FW, et al. Comment on acne and glycemic index. J Am Acad Dermatol 2008; 58: 175-7.

27. Ludwig DS. The glycemic index: physiological mechanisms relating to obesity, diabetes, and cardiovascular disease. JAMA 2002; 287: 2414-23.

28. Del Prete M, Mauriello MC, Faggiano A, et al. Insulin resistance and acne: a new risk factor for men? Endocrine 2012; 42: 555-60.

29. Stern MP. The insulin resistance syndrome. In: International textbook of diabetes mellitus. 2nd ed. Alberti KGMM, Zimmet P, De Fronzo RA, et al. John Wiley and Sons, Ltd. Chichester 1997; 255-83.

30. Kaymak Y, Adisen E, Ilter N, et al. Dietary glycemic index and glucose, insulin, insulin-like growth factor-1, insulin-like growth factor binding protein 3, and leptin levels in patients with acne. J Am Acad Dermatol 2007; 57: 819-23.

31. Melnik BC, John SM, Plewig G. Acne: risk indicator for increased body mass index and insulin resistance. Acta Derm Venereol 2013; 93: 644-9.

32. Matthews DR, Hosker JP, Rudenski AS, et al. Homeostasis model assessment: insulin resistance and beta-cell function from fasting plasma glucose and insulin concentrations in man. Diabetologia 1985; 28: 412-9.

33. DeFronzo RA, Tobin JD, Andres R. Glucose clamp technique: a method for quantifying insulin secretion and resistance. Am J Physiol 1979; 237: 214-23.

34. Bergman RN. Towards physiological understanding of glucose tolerance-minimal model approach. Diabetes 1989; 38: 1512-27.

35. Yeckel CW, Weiss R, Dziura J, et al. Validation of insulin sensitivity indices from oral glucose tolerance test parameters in obese children and adolescents. J Clin Endocrinol Metab 2004; 89: 1096-101.

36. Kurtoğlu S, Hatipoğlu N, Mazıcıoğlu M, et al. Insulin resistance in obese children and adolescents: HOMA-IR cut-off levels in the prepubertal and pubertal periods. J Clin Res Pediatr Endocrinol 2010; 2: 100-6.

37. Geloneze B, Vasques AC, Stabe CF, et al. HOMA1-IR and HOMA2-IR indexes in identifying insulin resistance and metabolic syndrome: Brazilian Metabolic Syndrome Study (BRAMS). Arq Bras Endocrinol Metabol 2009; 53: 281-7.

38. Arslan M. Guide of metabolic syndrome. Turkish Association of Endocrinology and Metabolism 2009; 1: 1-13. 